\title{
Maximization of the Annual Energy Production of Wind Power Plants by Optimization of Layout and Yaw-Based Wake Control
}

Pieter Gebraad

National Renewable Energy Laboratory

Jared Thomas

Brigham Young University, jaredthomas68@gmail.com

Andrew Ning

Brigham Young University, aning@byu.edu

Paul Fleming

National Renewable Energy Laboratory

Katherine Dykes

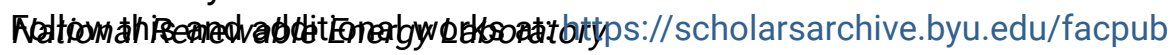

Part of the Mechanical Engineering Commons

\section{Original Publication Citation}

Gebraad, P., Thomas, J. J., Ning, A., Fleming, P., and Dykes, K., "Maximization of the Annual Energy Production of Wind Power Plants by Optimization of Layout and Yaw-Based Wake Control," Wind Energy, May 2016. doi:10.1002/we.1993

\section{BYU ScholarsArchive Citation}

Gebraad, Pieter; Thomas, Jared; Ning, Andrew; Fleming, Paul; and Dykes, Katherine, "Maximization of the Annual Energy Production of Wind Power Plants by Optimization of Layout and Yaw-Based Wake Control" (2016). Faculty Publications. 1739.

https://scholarsarchive.byu.edu/facpub/1739

This Peer-Reviewed Article is brought to you for free and open access by BYU ScholarsArchive. It has been accepted for inclusion in Faculty Publications by an authorized administrator of BYU ScholarsArchive. For more information, please contact ellen_amatangelo@byu.edu. 


\title{
Maximization of the annual energy production of wind power plants by optimization of layout and yaw-based wake control
}

\author{
Pieter M.O. Gebraad ${ }^{1}$, Jared J. Thomas ${ }^{2}$, Andrew Ning ${ }^{2}$, Paul A. Fleming ${ }^{1}$, and Katherine Dykes ${ }^{1}$ \\ ${ }^{1}$ National Renewable Energy Laboratory \\ ${ }^{2}$ Brigham Young University
}

\begin{abstract}
This paper presents a wind plant modeling and optimization tool that enables the maximization of wind plant annual energy production (AEP) using yaw-based wake steering control and layout changes. In order to make predictions of wind plant AEP, necessary extensions of the original wind plant wake interaction model include the coupling with a detailed rotor model and a control policy for turbine blade pitch and rotor speed. This coupling allows the prediction of power production with wake effects throughout a range of wind speeds. Results of an optimization study on a wind plant based on the Princess Amalia Wind Park show increases of 5\% in AEP by combined optimization of layout and wake steering control. Looking at the wind speed dependence of the possible power gains shows that power gains from wake steering control are highest in Region 1.5, and continue to be present to some extent for above-rated inflow speeds. The results show that layout optimization and wake steering are complementary to each other, in the sense that significant AEP improvements can be achieved with wake steering in a wind plant layout that is already optimized to reduce wake losses. Copyright @ 0000 John Wiley \& Sons, Ltd.
\end{abstract}

KEYWORDS

wind plant optimization; wind turbine control; wind turbine wakes; wind plant systems engineering

\section{INTRODUCTION}

Wind turbines in wind power plants impact each others performance through their wakes. This interaction reduces the total energy output of the plant. The negative effects of this aerodynamic interaction can be mitigated by placing the turbines further away from each other in the most prevalent wind directions. Therefore, plant design typically involves a plant layout optimization step where turbine placement is optimized to maximize the energy production by taking into account the effects of wakes [1]. In recent years, research has focused on improving power production of the wind plant by using coordinated control techniques between the turbines to mitigate the wake effects in the wind plant [2]. Recently, [3] proposed a combined optimization of wind plant layout and controls during the design phase, in order to realize a further increase of the power production of new wind plants. A limitation of many previous studies on wind plant wake control, including the combined optimization study of [3], is that only a single wind speed operating condition is considered, rather than taking into account the full range of wind plant operation conditions that a wind plant experiences throughout the year in order to maximize the annual energy production (AEP). This paper addresses this issue, and presents a wind plant optimization tool for the maximization of the AEP of the wind plant using the combination of wake control and layout changes.

The concept of wind plant wake control, which was first proposed in [4], is that the performance of the wind plant as a whole can be improved by coordinating the control operations across the wind turbines in such a way that wake losses are mitigated. Typically the wind plant controller coordinates the upstream turbines to reduce their power production in order to reduce the wake effects on downstream turbines, and increase the total power production of the wind plant. A review of the literature on different wake control strategies is found in [2]. Wake control techniques have different ways of using the turbine control degrees of freedom to affect the wakes. Most wind plant control studies in the literature use axial-induction-based control, in which generator torque or blade pitch is altered to optimize wake velocities. Alternatively, yaw offsets are used to redirect the wakes and steer them away from downstream turbines. In [5], we found that yaw-based 
wake steering control was the most effective of both approaches, based on high-fidelity simulations of the wind flow and wind turbine dynamics in a small turbine array using NREL's Simulator fOr Wind Farm Applications (SOWFA) [6].

For yaw-based wake steering control, Delft University of Technology and NREL developed an optimization strategy based on the FLOw Redirection and Induction in Steady-state (FLORIS) control-oriented model. FLORIS is a computationally efficient model that predicts the steady-state wake characteristics, and the resulting power production of each turbine in the wind plant, as a function of the yaw angles and the positions of the turbines. The model can be used to maximize the power production of wind plants by predicting the optimal yaw angles. In [7], the FLORIS-based yaw control approach has been successfully tested in an online implementation in SOWFA. In [3], the FLORIS model was used in a wind plant system engineering approach with combined optimization of yaw control and wind plant layout. It was found that a further increase of the power density (generated electrical power per land area) of new wind plants can be achieved through this combined optimization. This can lead to reduced costs for the rest of the plant, including the costs of roads, electric cables, and land leasing costs.

The previous work [7] and [3] on optimization of wake steering only considered a single wind speed of the inflow of the wind farm. This is because the FLORIS wake model was only suited to predict the operation in the Region 2 control operating point of the wind turbine, with a fixed blade pitch angle and tip-speed ratio. In order to predict and optimize the Annual Energy Production (AEP) of the wind plant with specific wind resource characteristics consisting of both a wind speed and a wind direction distribution, the wind plant model needs to be suited to predict the power output of the wind plant for the full range of wind speeds in which the wind plant operates.

To arrive at such a model describing the full wind plant operational range, in this paper we couple the FLORIS wake model with a more comprehensive rotor model, and we include the power and speed control policy of the turbine. The rotor model consist of the WISDEM CCBlade blade element momentum (BEM) code [8], and the coupling of the models is performed through the WISDEM framework for wind plant modeling. WISDEM (Wind-Plant Integrated System Design and Engineering Model) [9] is a set of models for assessing overall wind plant cost and production, that is built using the Open-source platform for Multidisciplinary Design, Analysis, and Optimization (OpenMDAO) [10]. We use efficient gradient-based optimization techniques available in the WISDEM toolset, to perform an optimization of AEP in a case study on a full size wind plant using yaw control and layout changes. We discuss in detail how the rotor characteristics combined with the power and speed control policy affect the power production increases that can be achieved through yaw-based wake steering control.

In section 2 we introduce the objective and optimization variables of the wind plant AEP optimization problem considered in this paper. In section 3 we present the new WISDEM FLORIS wind plant AEP model. More details on the optimization strategies and tools used to solve the AEP optimization problem are provided in section 4 . In section 5 we present the optimization case study on a full size wind plant (based on the Princess Amalia Wind Park). Finally, we discuss our conclusions in section 6 .

\section{OPTIMIZATION OBJECTIVE AND VARIABLES}

In this study, we focus on the problem of optimizing the AEP of a wind plant for a given site with layout constraints (a bounded area in which turbines can be placed, and a minimum spacing between the turbines) and with an expected wind distribution for the site, using the set-points for the yaw angles and the positions of the turbines as the optimization variables. While the positions of the turbines are fixed, the yaw set-point will be adjusted to wind speed and direction.

The wind distribution will be described by the wind rose, which consists of a two-dimensional histogram of the wind speed and wind direction with a fine resolution (i.e. a large number of bins). The center wind speed and direction of each bin $b$ in the wind rose are respectively denoted as $V_{b}$ and $\phi_{b}$. The frequency of occurence of each bin is written as $p_{b}$, and expresses the portion of annual hours, i.e. $0 \leq p_{b} \leq 1$. We denote the number of bins in the wind rose as $N_{B}$.

The optimization variables considered are the location $\left(x_{t}, y_{t}\right)$ of each turbine $t$, and the yaw angles of each turbine for each wind speed and direction bin, $\gamma_{b, t}$. The number of turbines in the wind plant is denoted as $N_{T}$.

Using the shorthand notation $\left\{a_{i}\right\}_{i=1}^{N}$ for the set of variables $a_{1}, \ldots, a_{N}$, the optimization problem can be written as follows:

$$
\underset{x, y, \gamma}{\operatorname{maximize}} \operatorname{AEP}\left(\left\{x_{t}, y_{t}\right\}_{t=1}^{N_{T}},\left\{\left\{\gamma_{b, t}\right\}_{t=1}^{N_{T}} ; V_{b}, \phi_{b}, p_{b}\right\}_{b=1}^{N_{B}}\right) \quad \text { subject to layout constraints }
$$

The AEP is calculated as the weighted sum of the total wind plant power production $P_{b}$ for each wind rose bin $b$. The weighting is given by the frequency of each bin, $p_{b}$, times the number of hours in the year, $N_{h}=8760$ :

$$
\mathrm{AEP}=\sum_{b=1}^{N_{B}} N_{h} P_{b}\left(\left\{x_{t}, y_{t}\right\}_{t=1}^{N_{T}},\left\{\gamma_{b, t}\right\}_{t=1}^{N_{T}} ; V_{b}, \phi_{b}\right) p_{b}
$$


The control variables of the wind turbine other than the yaw angle, typically blade pitch and generator torque, are not considered optimization variables here. They are set using a fixed turbine power and rotor speed control policy. In this optimization scheme, we assume that the yaw control is set by a plant-level control that uses wake steering to minimize the wake losses and maximize energy production, while the power and speed controller operates on the individual turbine level, and adjusts the pitch and generator torque such that the turbine is operating within certain limits. For example, it assures that the turbine's rotor speed and power are at or below their rated levels for each wind speed and yaw setting. The coupling of each of the submodels in the wind plant model will be discussed in the next Section 3.

\section{WIND PLANT MODELING}

In this section we discuss the wind plant model that is used to predict and optimize the wind plant AEP. It consists of the FLORIS wake model coupled with other WISDEM submodels.

The FLORIS wake model was presented in [7]. It is a combination of an extended Jensen wake model [11] and a model for wake deflection caused by yaw offsets proposed by Jiménez et al. [12]. The Jensen model, which uses a uniform velocity deficit with a top-hat profile that decays and widens with downstream distance, was augmented by including several wake zones, each with their own expansion and recovery properties, to better model situations with partial wake overlap, which is relevant for yaw-based wake control. The FLORIS model is a parametric model for which the parameters are tuned based on measured data or data from high-fidelity simulations. The full description of the original FLORIS model in [7] includes a detailed example of tuning FLORIS based on high-fidelity comput simulation data generated by the SOWFA tool.

The original FLORIS presented in [7] included a highly simplified characterization of the rotor that assumed a constant axial-induction factor $a=1 / 3$, and idealized relationships between the axial-induction factor and the thrust and power coefficients. Influences of the tip-speed ratio and pitch were not included, and therefore the rotor model was only applicable in region 2 when the turbine operates at peak efficiency with a constant pitch and tip-speed ratio.

In order to model the AEP of a wind plant with wake steering control, we use the WISDEM modeling framework to couple the FLORIS wake model to a BEM code that provides the thrust and power characteristics of the wind turbine rotors for all operating points, and to a model of the power and speed controller of the turbine. This enables the model to make predictions of power production for the full range of wind speeds in which the wind plant operates.

\subsection{Overview of the simulation and optimization scheme}

An overview of the coupled model scheme for wind plant AEP simulation and optimization is given in Figure 1. The chart was created using the XDSM (eXtended Design Structure Matrix) standards [13]. The main diagonal contains all the components. Inputs and outputs are connected to the relevant submodels by thick gray lines. Inputs to components are shown in the same column. Outputs from components are shown in the same row. System inputs are listed in the first row, system outputs are to the left of the first column. Ovals represent optimization algorithms or solvers, rectangles represent system components. The flow of execution is shown by the thin black lines. The "stacked" labels indicate parallel execution.

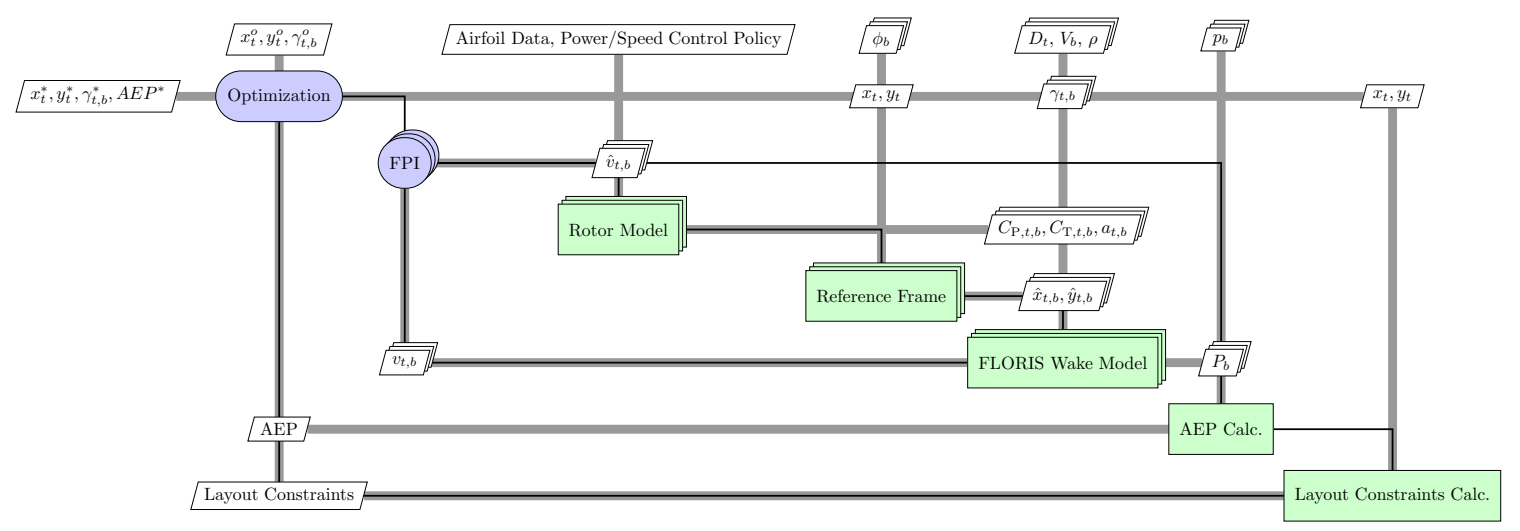

Figure 1. XDSM chart of the WISDEM FLORIS wind plant AEP modeling and optimization tool. 
Like in previous equations (1) and (2), in the model scheme we use the indexing $(\cdot)_{t}$ for a variable that applies to a turbine $t$ for all wind rose bins, and $(\cdot)_{t, b}$ for a turbine variable that depends on the bin $b$ that the wind plant is operating in. The starting value of each optimization variable is indicated as $(\cdot)^{\circ}$, and the final solution as $(\cdot)^{*}$.

As explained in the previous section, the optimization algorithm maximizes the total AEP of the wind plant by optimizing the yaw angles $\gamma_{t, b}$ and the positions of the turbines $x_{t}, y_{t}$, with some predefined constraints on the layout. In each iteration of the optimization, the model calculates the power of each turbine in the wind plant for each bin $b$ in the wind rose, with a center wind direction $\phi_{b}$ and center free-stream wind speed $V_{b}$, and sums up the turbine powers to get total wind plant power production $P_{b}$ for each wind rose bin $b$. The calculation of the total power $P_{b}$ for each bin $b$ can be performed in parallel. After that, the AEP is calculated through equation (2).

In order to estimate the powers of the turbines, the FLORIS wake model needs the air density $\rho$, the turbine yaw angles $\gamma_{t, b}$, rotor diameters $D_{t}$, and positions in the downwind-crosswind reference frame $\hat{x}_{t, b}, \hat{y}_{t, b}$ (see section 3.2 of [7]). Also, the wake model needs the power and thrust coefficients $C_{\mathrm{P}, t, b}, C_{\mathrm{T}, t, b}$ and the axial-induction factor $a_{t, b}$ of each turbine, which are supplied by the rotor model. The power coefficient $C_{\mathrm{P}, t, b}$ determines the efficiency with which the wind kinetic power is converted to electrical power by the rotor, and the thrust coefficient $C_{\mathrm{T}, t, b}$ then determines the reduction of velocity over the rotor plane (represented by the axial-induction factor, $a_{t, b}$ ) to the initial velocity of the wake just behind the rotor. The rotor model is in a feedback loop with the wake, which is solved using OpenMDAO's fixed-point iterator (FPI) that iterates on the effective wind speed at each turbine, $v_{t, b}$, as will be explained in more detail in the next section.

\subsection{The rotor model}

The rotor model is based on the WISDEM CCBlade BEM code [8] calculating the power coefficient $C_{\mathrm{P}}$ and the thrust coefficient $C_{\mathrm{T}}$ of the rotor based on the wind speed. The rotor model also includes the possible influences of the pitch and rotor speed operating point set by the power and speed control policy, and the yaw angle of each rotor. In our model scheme in Figure 1, the yaw angle is an external input to the rotor model, that is set by the optimizer. The different parts of the rotor model are coupled in series, as follows:

- First, $V_{o}$, the component of the rotor-effective wind speed $V$ that is orthogonal to the possibly yawed rotor plane, is calculated, through $V_{o}=V \cos (\gamma)$, where $\gamma$ is the yaw angle of the rotor.

- A wind turbine power and speed control policy is applied, that sets the collective blade pitch and rotor speed as a function of the orthogonal wind speed component $V_{o}$ (see Figure 2, for example). Note that this is an abstraction of the steady-state behavior of a real controller (which generally uses no knowledge of the wind speed directly).

- The WISDEM CCBlade BEM code generates the $C_{\mathrm{P}}$ - and $C_{\mathrm{T}}$ - coefficients of the rotor based on the wind speed, yaw angle, rotor speed, and blade pitch angle.

- Finally, the axial-induction factor is calculated from the $C_{\mathrm{T}}$-factor based on rotor theory with the Glauert correction: [14]:

$$
a= \begin{cases}0.143+\sqrt{0.0203-0.6427\left(0.889-C_{\mathrm{T}}\right)} & \text { if } C_{\mathrm{T}}>0.96 \\ \frac{1}{2}\left(1-\sqrt{1-C_{\mathrm{T}}}\right) & \text { else. }\end{cases}
$$

The combination of these parts yields the turbine's power and thrust coefficient surfaces as a function of wind speed and yaw, shown in Figure 3.

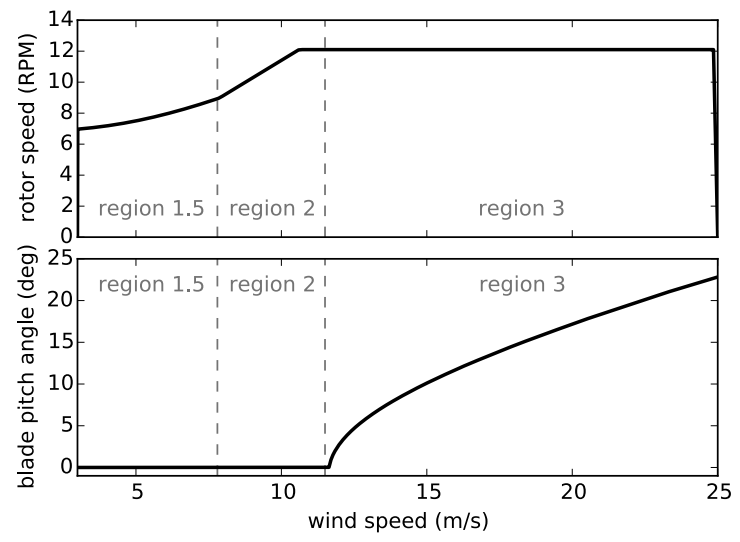

Figure 2. Blade pitch and rotor speed control policy of the NREL 5-MW reference wind turbine as applied in the rotor model, based on the controller description in [15]. 


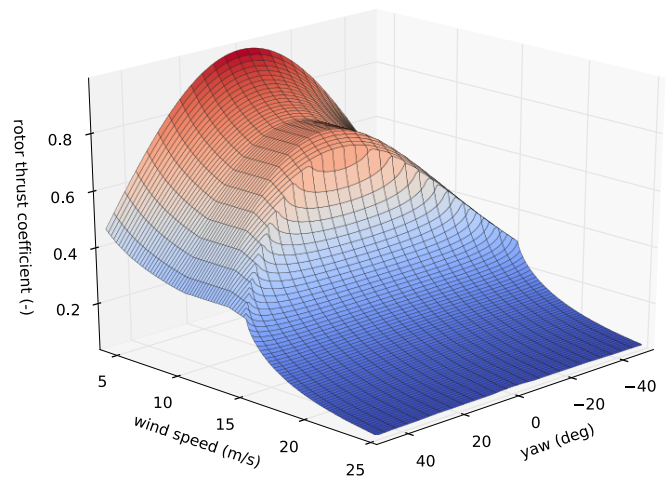

(a)

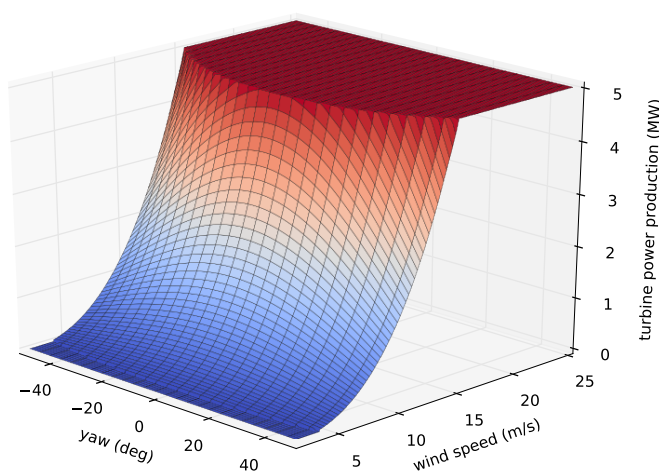

(b)

Figure 3. Thrust coefficient and power production of the NREL 5-MW reference wind turbine as a function of yaw and effective wind speed, as predicted by the rotor model

The wake model uses the thrust coefficients $C_{\mathrm{T}}$ and the axial-induction factor $a$, and the yaw angles $\gamma$ to calculate the wake velocity deficit and redirection properties, and then predicts the effective wind speeds $v$ and power production at the downstream turbines based its the wake recovery and deflection assumptions. Because the rotor model needs the rotor-effective wind speed $v$ as an input, the effective velocities $v$ calculated by the wake model are fed back into the rotor models for the downstream turbines (see Figure 1). The rotor-effective wind speeds therefore have to be found through an iterative procedure, for which we use the FPI.

By coupling the rotor characteristics with the FLORIS wake model, FLORIS has an improved ability to predict wakes and power production in above-rated operation ("region 3") and in low-wind start-up conditions ("region 1.5"). Between those regions, in region 2, an optimal tip-speed ratio and zero pitch is used for maximum power extraction. For above-rated operation in high wind speed conditions in region 3, the rotor speed is held to its rated value, and pitch offset control is used to shed loads and maintain rated power. In this region, the lower thrust coefficient of the rotor (see Figure 3a) results in a smaller wake velocity deficit (relative to the free-stream velocity) than in the strong wake that is generated when the turbine is operating at the peak efficiency in region 2. Region 1.5 is a transition between region 1 (operation at or below cut-in wind speed, where the turbine accelerates for start-up) and region 2. In the NREL 5-MW turbine, this region is used to place a lower limit on the generator speed. The thrust coefficient in region 1.5 is higher than in other regions because the tip-speed ratio is higher, which results in a deeper relative velocity deficit in the wake.

To improve computational efficiency during optimization, the power and thrust coefficient surfaces (as shown in Figure 3) are pre-calculated on a fine mesh for the considered turbine, and the simulation of the rotor model in the simulation and optimization scheme consists of interpolation of these precalculated surfaces. This was found to be more computationally efficient than evaluating the CCBlade model in combination with controls in each iteration of the FPI.

\subsection{Adjustment of the FLORIS wake model parameters}

Because the FLORIS wake model is coupled with a new rotor model, different (more realistic) values for the axialinduction, $C_{\mathrm{P}}$, and $C_{\mathrm{T}}$ factors are fed into the wake model. Therefore some of the parameters of the FLORIS wake model have to be updated from the values reported in [7], to provide the same fit with high-fidelity CFD simulation cases in SOWFA. By following the same tuning procedure as described in [7], based on the same SOWFA cases with yaw offsets and re-positioning of turbines, the wake model parameters for the new coupled FLORIS model are found. The new parameters are listed in Table I.

\begin{tabular}{|ll|ll|llll|}
\hline \multicolumn{2}{|c|}{ wake deflection } & \multicolumn{3}{|c|}{ wake expansion } & \multicolumn{4}{|c|}{ wake velocity recovery } \\
\hline$k_{d}$ & $\mathbf{0 . 1 7}$ & $k_{e}$ & $\mathbf{0 . 0 5}$ & $M_{U, 1}$ & 0.5 & $a_{U}$ & $\mathbf{1 2 . 0}$ \\
$a_{d}$ & -4.5 & $m_{e, 1}$ & -0.5 & $M_{U, 2}$ & 1 & $b_{U}$ & $\mathbf{1 . 3}$ \\
$b_{d}$ & -0.01 & $m_{e, 2}$ & 0.22 & $M_{U, 3}$ & 5.5 & & \\
& & $m_{e, 3}$ & 1 & & & & \\
\hline
\end{tabular}

Table I. Wake model parameters for the new FLORIS model. Parameter values that are different from the values reported in [7], are in bold. 


\section{OPTIMIZATION STRATEGY AND TOOLS}

Solving the AEP optimization problem for a full size wind plant is a large optimization problem. This section discusses the steps and tools used to solve the problem.

\subsection{Optimization steps}

The number of optimization variables in optimization problem (1) is $N_{T} * N_{B}+2 * N_{T}$. The number of bins $N_{B}$ in the wind rose is the product of number of wind direction bins $\left(N_{D}\right)$ and the number of wind speed bins $\left(N_{S}\right)$. In order to have a reliable estimate of the AEP, a fine binning in the wind rose has to be used. This results in a large number of optimization variables. Therefore, in order to simplify the optimization problem somewhat, We have split up the optimization in three steps:

1. We first perform the layout only where the AEP calculation is based on a simplified wind rose with one averaged wind speed for each wind direction, such that $N_{B}$ reduces to $N_{D}$.

2. We then perform the combined optimization of layout and yaw where the AEP calculation is again based on one averaged wind speed for each wind direction.

3. We perform the optimization of yaw angles for each wind speed and direction bin of the full wind rose separately, without further optimizing the positions. This step consists of solving $N_{B}=N_{D} * N_{S}$ separate optimization problems with only $N_{T}$ optimization variables.

The layout optimization in step 1 is used to "warm start" the coupled layout/yaw optimization in step 2, and similarly step 2 provides a starting point for finding the full set of yaw angles for each wind direction and speed in step 3. It was found that warm starting with a better starting point significantly improved convergence speed. In the case study in Section 5 , we confirm that each step will result in an increase in AEP calculated based on the full wind rose.

\subsection{Gradient-based optimization}

There are two main divisions of optimization methods: gradient-free and gradient-based. While many wind plant layouts are optimized using gradient-free approaches, gradient-based optimization is preferred for the problem considered in this paper because of its ability to reliably handle large numbers of design variables while still accurately converging. The optimization is performed using SNOPT, a gradient-based sequential quadratic programming optimization algorithm for solving large, nonlinear problems with constraints [16].

As was pointed out in [17], wake models with discontinuous functions describing the wake velocity profile, such as the FLORIS model, are less suited for large-scale gradient-based optimization than wake models with smooth functions. Therefore, we have performed local smoothing on the discontinuities in the wake definitions in the FLORIS model. The alterations to the model do not change the results for a turbine spacing greater than one rotor diameter, and therefore have no effect on the final result of an optimization with minimum spacing constraints, but they can simplify the definition of the gradients of the model and improve convergence properties in the optimization. More explanations on these smoothing adaptations are provided in [18].

Also, in order to prevent finite differencing approximation errors and inefficiencies, we provided the exact analytic gradients of the FLORIS wake definitions. These analytic gradients were found from the FLORIS code using the Tapenade automatic differentiation tool [19].

\section{CASE STUDY: RE-DESIGN AND WAKE CONTROL FOR THE PRINCESS AMALIA WIND PARK}

In this case study, we perform a re-design of the Princess Amalia Wind Park (PAWP), an offshore wind plant in the North Sea consisting of 60 turbines, which is located $23 \mathrm{~km}$ off the coast of the Netherlands. The optimization study was performed using distributed computing on NREL's Peregrine high-performance computing system [20].

The wind rose that is used as a basis for the re-design is generated from public data available in [21] from wind measurements at a nearby location in the North Sea. These wind measurements were made by the NoordZeeWind meteorological mast [22] during the period from July 1, 2005 to June 30, 2006* The measurements consist of 10-minute averages of the wind direction and wind speed. From these measurements we generate the wind rose that is used to calculate the AEP, which shown in Figure 4. The wind rose has a wind direction bin width of $5^{\circ}$ and a wind speed bin width of 1 $\mathrm{m} / \mathrm{s}$ throughout the operational range of the turbine (between 3 and $25 \mathrm{~m} / \mathrm{s}$ ).

\footnotetext{
* These measurements are undisturbed by the NoordzeeWind-offshore wind plant that was constructed later.
} 


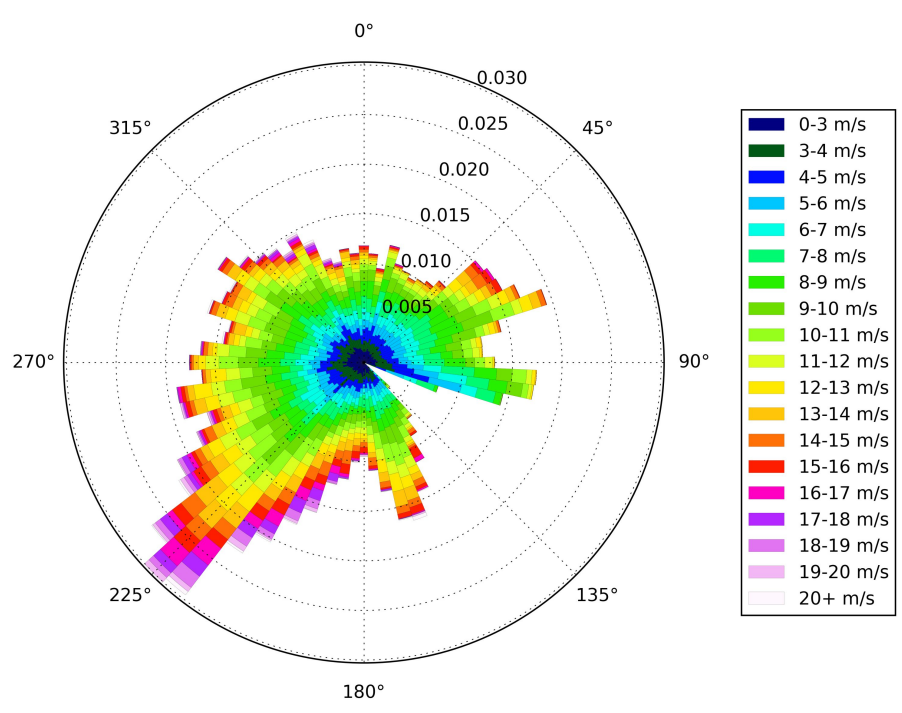

Figure 4. The wind rose used to calculate the AEP in the PAWP optimization case study. In this picture wind speed bins above 20 $\mathrm{m} / \mathrm{s}$ are lumped together for better visibility, but in the wind rose used for the study, the wind rose is binned with a $1 \mathrm{~m} / \mathrm{s}$ resolution up to $25 \mathrm{~m} / \mathrm{s}$.

In the re-design of the wind plant we use NREL 5-MW reference turbines instead of the Vestas V80-2MW machines used in the real PAWP. The NREL 5-MW turbine has a larger rotor diameter of $D=126 \mathrm{~m}$ than the V80-2MW turbine $(D=80 \mathrm{~m})$. Placing the NREL 5-MW turbines at the same positions as in the original PAWP results in a turbine spacing between neighboring turbines of $4.4 \mathrm{D}$ or more, instead of the minimum $6.9 \mathrm{D}$ spacing in the real PAWP.

We initially only optimize the layout of the wind plant (step 1 in Section 4.1), taking the initial layout of the PAWP as a starting point of the gradient-based search. We use layout constraints consisting of a minimum $2 D$ spacing between the turbines, and the restriction that the turbines are in a convex hull surrounding the original layout. ${ }^{\dagger}$ Hence, in this optimization problem we maximize AEP for a given area of land by changing the layout. We thereby neglect other factors that may impact the cost of energy such as cable length and the needed tower height considering water depth, and practical constraints such as ship maneuverability between the turbines. After the initial layout optimization, a combined optimization of layout and yaw is performed based on average wind speed per wind direction (step 2), and finally, with the result of the combined optimization as a starting point, optimization of the yaw angles for each wind direction and wind speed are performed (step 3). It should be noted that the combined optimization in step 2 does not change the layout much from the result of step 1 (the largest turbine displacement is $0.27 D$ ). Improvements in this step are thus mostly a result of wake steering yaw control.

\begin{tabular}{|r|l|l|l|l|}
\cline { 2 - 5 } \multicolumn{1}{c|}{} & Original layout & $\begin{array}{l}\text { Optimized layout, } \\
\text { zero yaw }\end{array}$ & $\begin{array}{l}\text { Optimized layout, yaw } \\
\text { adjusted to wind direction }\end{array}$ & $\begin{array}{l}\text { Fully optimized layout } \\
\text { and yaw }\end{array}$ \\
\hline AEP & $1040.3 \mathrm{GWh}$ & $1055.8 \mathrm{GWh}$ & $1094.0 \mathrm{GWh}$ & $1095.0 \mathrm{GWh}$ \\
\hline AEP increase & (baseline) & $1.5 \%$ & $5.2 \%$ & $5.3 \%$ \\
\hline Wake Losses & $14.4 \%$ & $13.1 \%$ & $10.0 \%$ & $9.9 \%$ \\
\hline Reduction of Wake Losses & (baseline) & $8.8 \%$ & $30.7 \%$ & $31.4 \%$ \\
\hline
\end{tabular}

Table II. AEP Optimization Results

The AEP results of the optimization steps are listed in Table II. For the final layout without wake steering (i.e. yaw offsets of zero degrees), an improvement of $1.5 \%$ of AEP is achieved over the original layout. The original layout already suits the wind conditions quite well, with a large spacing in the dominant wind direction (Southwest), and therefore only a relatively small improvement was to be expected from the layout optimization. Alternatively, we can compare each case with a case without production losses from wakes (the AEP of 60 turbines operating in isolation, with the same wind

\footnotetext{
${ }^{\dagger}$ The minimum spacing constraint results in a quadratic inequality constraint, and the convex outer boundary results in a linear inequality constraint. For both constraints, we provided the analytic gradients to the optimizer.
} 
speeds as in the wind rose). We then find that in the original layout, wake losses amount to $14.4 \%$, which are reduced to $13.1 \%$ by optimizing the layout further. If we look at the improvements attained by wake steering, we see that adjusting yaw settings to wind direction yield an increase of AEP of $3.7 \%$, and a reduction of wake losses of $24.4 \%$ compared to the optimized layout without wake steering yaw offsets. Combining the improvements of layout and yaw adjustments to wind direction (the result of step 2) yields an increase of AEP of 5.2\% over the original layout without wake steering. Wake losses are reduced to $10.0 \%$. Only a small increase in AEP is obtained by further adjusting the yaw angles to wind speed in step 3, and finally the total optimization procedure increases the AEP by $5.3 \%$ and reduces wake losses to $9.9 \%$.

Figure 5 shows the total wind plant power production for the optimized layout without wake for each wind direction and speed (left subplot), and the power increases that can be achieved by optimizing the yaw angles for each wind direction and speed (middle subplot). The right subplot shows the difference in wind plant power with the result of optimization step 2 , i.e. the case where yaw angles are adjusted to wind direction only using the average wind speed for that direction. In the middle plot, it can be seen that the power improvements that can be achieved with wake steering are varying strongly with wind direction. This is because higher gains are achieved in the wind directions with close inter-turbine spacings. Checking the relationship to speed, improvements occur from cut-in wind speeds up until $15 \mathrm{~m} / \mathrm{s}$. That no improvements occur in the highest wind speeds is logical, given that with these wind speeds even waked turbines are producing rated power and therefore additional improvement is not possible. Note that even though the NREL 5-MW turbine is rated at $11.4 \mathrm{~m} / \mathrm{s}$, the improvements continue above rated inflow wind speeds, so long as some of the waked downstream turbine are below rated in the zero yaw case. The improvements peak around $6 \mathrm{~m} / \mathrm{s}$. Figure 6 shows the FLORIS-predicted flow fields for a case with a $5.5 \mathrm{~m} / \mathrm{s}$ inflow speed, which is illustrative for these low wind speed cases, for several steps in the optimization. For these cases, the front turbines are operating in region 1.5, which makes the initial wake losses larger because of high rotor thrust levels, as explained in section 3.2, rasing the potential for improvement with wake steering. Also for these low wind speeds wake steering on the front turbines can "push" the wind speed at some of the back turbines above the cut-in wind speed. This makes that for these lower wind speeds, waked turbines that produce no power in the zero yaw case, will start producing when wake steering is enabled.

For the same reason, the right subplot in Figure 5 shows that the adjustment of the yaw angles to both wind speed and direction, rather than to wind direction and the average wind speed for that wind direction only, has most benefit for low wind speeds (up to 7.8\%). Within region 2 there are no improvements from adjusting yaw to wind speed. This is because for each of the displayed wind directions, the average wind speed is within region 2, and within region 2 the optimal yaw angles do not change because the thrust coefficients of the turbines remain constant. Small differences, up to $1.5 \%$, are seen for above-rated wind speeds, where upstream turbines can have a yaw misalignment without going below the rated power, and thus larger yaw angles are used for wake steering than for wind speeds in region 2. Although the power differences are significant, the fact that they exist in a limited band of non-frequently occurring wind speeds, explains why the improvement in AEP from adjusting yaw to wind speed is small (from 5.2\% to 5.3\% as shown in Table II).

The 5.3\% AEP increase reported in Table II is of the same order as the 8.6\% power production increase that was reported in [3] for a similar optimization based on the PAWP that considered inflow conditions with $8 \mathrm{~m} / \mathrm{s}$ wind speed only. The fact that for higher wind speeds the power improvements are lower, explains why the AEP increase is lower than $8.6 \%$. The results on AEP increases are more insightful for wind plant system engineering, because they represent the full range of annual operating conditions of the wind plant.

\section{CONCLUSION}

This paper presented the WISDEM FLORIS wind plant modeling and simulation tool, which combines different submodels to enable the optimization of wind plant AEP. Necessary extensions of the original FLORIS wake model included the coupling with a more detailed rotor model and a control policy for pitch and rotor speed, that allowed the prediction of power production with wake effects throughout a range of wind speeds. We used gradient-based optimization tools available in the WISDEM tool set to enable large-scale gradient-based optimization techniques based on exact and smooth gradients of the wake model.

Results of an optimization study on the Princess Amalia Wind Park showed significant increases in AEP by using wake steering with yaw control. An AEP increase of 5.3\% was achieved through combined optimization of yaw and layout. The results show that layout optimization and wake steering are complementary to each other, in the sense that a significant AEP improvement $(3.7 \%)$ is achieved by wake steering in a wind plant layout that is already optimized to reduce wake losses.

Looking at the wind speed dependence of the possible power gains of wake steering, shows that power gains are highest in region 1.5, and that smaller relative power improvements continue to be present in above-rated wind speeds.

It is important to point out that the predicted AEP improvements should be considered, most-likely, an upper bound on expected performance. Effects not captured in the wind plant model, such as dynamic wind direction changes, will likely limit to some extent the ability to realize the projected improvements through wake steering. However, there is 

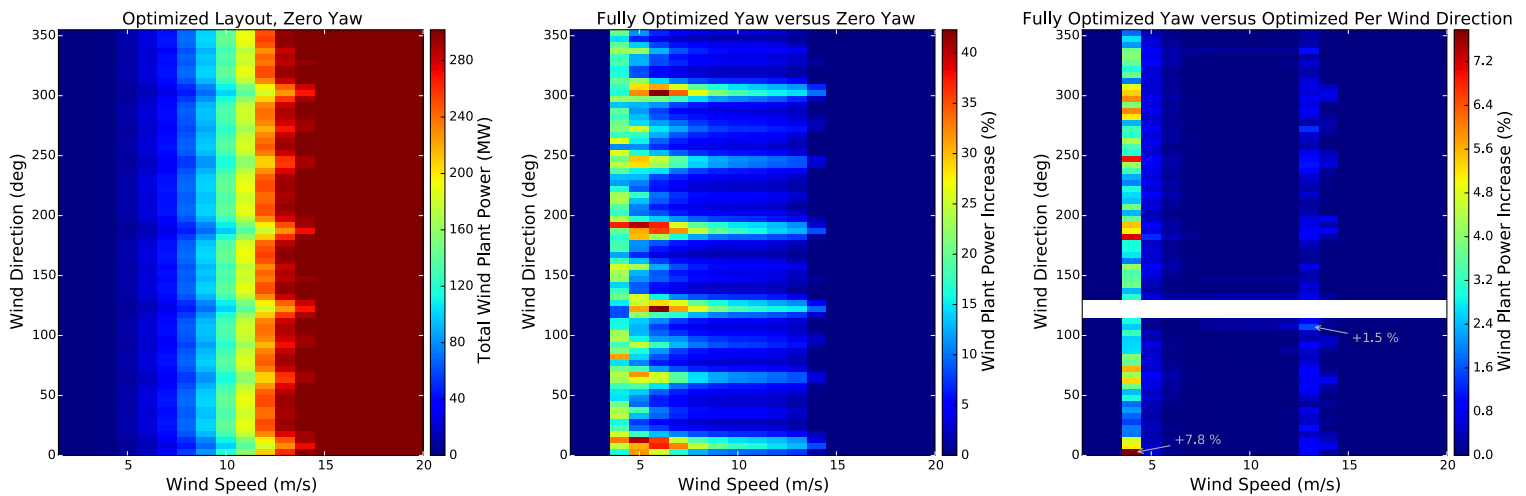

Figure 5. The left plot shows the total power production of the wind plant with optimized layout, as a function of wind direction and wind speed for the case without wake steering. The center plot shows the increase in wind plant power when wake steering is used, when the yaw angles are adjusted to both wind speed and direction. The right plot shows the increases of power of this fully optimized wake steering case compared to the case when the yaw angles are only adjusted to wind direction. Wind directions with an average wind speed that is below cut-in wind speed of the turbines, are excluded from the right subplot.
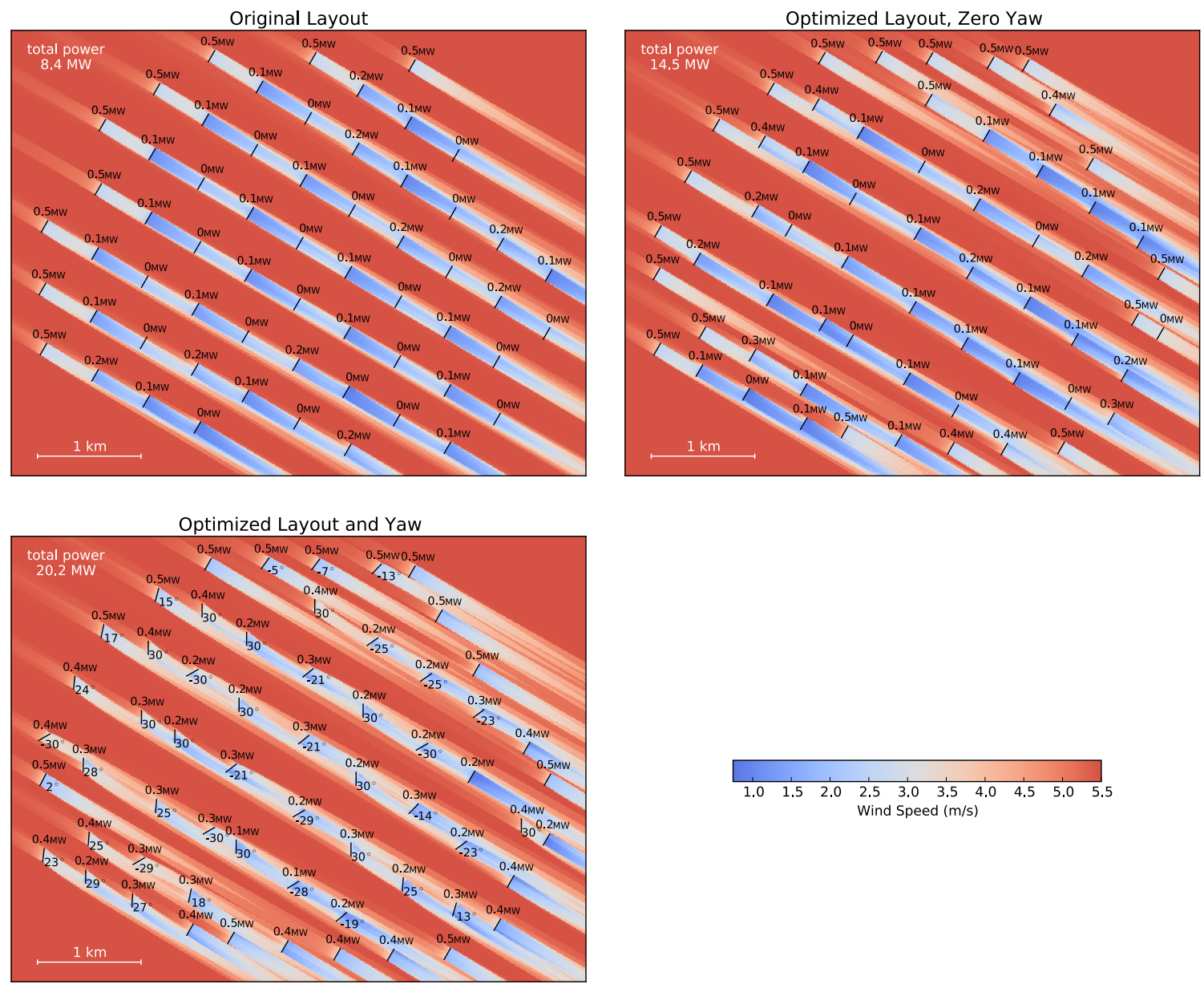

Figure 6. Hub-height flow field as predicted by FLORIS model for a free-stream wind speed of $5.5 \mathrm{~m} / \mathrm{s}$ and a $300^{\circ}$ wind direction, for the original layout, and two further optimized cases. 
also the possibility that improvements to technology will again raise this potential. Possibilities to extend the benefits of wind plant wake steering control using a system engineering approach include redesigning the rotor blades for improved wake control, and redesigning the power and speed controller taking into account the wake effects. Also, this study only investigated AEP improvement, however, ongoing research seeks to maximize the benefit of wind plant control to cost of energy through active control that responds to grid demands [23], and reduces loads.

\section{ACKNOWLEDGMENTS}

This work was supported by the U.S. Department of Energy under Contract No. DE-AC36-08GO28308 with the National Renewable Energy Laboratory. Funding for the work was provided by the DOE Office of Energy Efficiency and Renewable Energy, Wind and Water Power Technologies Office.

\section{REFERENCES}

1. Herbert-Acero JF, Probst O, Réthoré PE, Larsen GC, Castillo-Villar KK. A review of methodological approaches for the design and optimization of wind farms. Energies 2014; 7(11):6930-7016.

2. Knudsen T, Bak T, Svenstrup M. Survey of wind farm control - power and fatigue optimization. Wind Energy 2014; .

3. Fleming PA, Ning A, Gebraad PMO, Dykes K. Wind plant system engineering through optimization of layout and yaw control. Wind Energy 2015; .

4. Steinbuch M, de Boer WW, Bosgra O, Peters S, Ploeg J. Optimal control of wind power plants. Journal of Wind Engineering and Industrial Aerodynamics 1988; 27:237-246.

5. Gebraad PMO, Fleming PA, van Wingerden JW. Comparison of actuation methods for wake control in wind plants. American Control Conference, Chicago, USA, 2015.

6. Churchfield M, Lee S. National Wind Technology Center information portal - SOWFA. https: / / nwtc.nrel.gov/SOWFA 2015.

7. Gebraad PMO, Teeuwisse FW, van Wingerden JW, Fleming PA, Ruben SD, Marden JR, Pao LY. Wind plant power optimization through yaw control using a parametric model for wake effects - a CFD simulation study. Wind Energy $2014 ;$.

8. Ning A. National Wind Technology Center information portal - CCBlade. https: / / nwtc.nrel.gov/CCBlade 2015.

9. Dykes K, Ning A, Graf P, Scott G, Guo G, King R, Parsons T, Damiani R, Fleming P. National Wind Technology Center information portal - WISDEM. https : / / nwt c. nrel.gov/WISDEM 2015.

10. NASA Glenn Research Center. An open-source MDAO framework written in Python. http: / / www. openmdao.org.

11. Jensen NO. A note on wind generator interaction. Technical Report Ris $\phi$-M-2411, Ris $\varnothing$ National Laboratory 1984.

12. Jiménez A, Crespo A, Migoya E. Application of a LES technique to characterize the wake deflection of a wind turbine in yaw. Wind Energy 2010; 13(6):559-572.

13. Lambe AB, Martins JRRA. Extensions to the design structure matrix for the description of multidisciplinary design, analysis, and optimization processes. Structural and Multidisciplinary Optimization 2012; 46(2):273-284.

14. Manwell JF, McGowan JG, Rogers AL. Wind Energy Explained - Theory, Design and Application, chap. 3.7 General Rotor Blade Shape Performance Prediction. John Wiley \& Sons, 2002; 120-121.

15. Jonkman J, Butterfield S, Musial W, Scott G. Definition of a 5-MW reference wind turbine for offshore system development. Technical Report NREL/TP-500-38060, National Renewable Energy Laboratory 2009.

16. Gill PE, Murray W, Saunders MA. SNOPT: An SQP algorithm for large-scale constrained optimization. SIAM journal on optimization 2002; 12(4):979-1006.

17. Thomas J, Tingey E, Ning A. Comparison of two wake models for use in gradient-based wind farm layout optimization. IEEE Conference on Technologies for Sustainability, Ogden, UT, USA, 2015.

18. Thomas J, Gebraad P, Ning A. Changes to the FLORIS model to improve the wake definition and ensure continuity and differentiability. Unpublished 2015; .

19. Hascoet L, Pascual V. The Tapenade automatic differentiation tool: principles, model, and specification. ACM Transactions on Mathematical Software May 2013; 39(3):20:1-20:43, doi:10.1145/2450153.2450158. URL http://doi.acm.org/10.1145/2450153.2450158.

20. National Renewable Energy Laboratory. NREL's high-performance computing capabilities. http: / /hpc.nrel.gov 2015.

21. NoordzeeWind BV. Data of the NoordzeeWind monitoring and evaluation programme (NSW-MEP). http://www. noordzeewind.nl/en/knowledge/reportsdata/. 
P.M.O. Gebraad et al. Maximization of the annual energy production of wind power plants by optimization of layout and wake control

22. Brand AJ, Wagenaar JW, Eecen PJ, Holtslag MC. Database of measurements on the Offshore Wind Farm Egmond aan Zee. Proceedings of the EWEA Annual Meeting, Copenhagen, Denmark, 2013.

23. Fleming P, Aho J, Gebraad P, Pao L, Zhang Y. CFD simulation study of active power control in wind plants. Submitted to American Control Conference, 2016. 